\title{
A novel mutation of thyroid hormone receptor $\beta$ in exon 10 in a case of thyroid hormone-resistant non-Hodgkin's lymphoma of the thyroid
}

\author{
KE CHEN, YANHONG XIE, LILING ZHAO, SHAOLI ZHAO, HONGHUI HE and ZHAOHUI MO \\ Department of Endocrinology, Third Xiangya Hospital of Central South University, Changsha, Hunan 410013, P.R. China
}

Received February 17, 2014; Accepted October 29, 2014

DOI: $10.3892 / \mathrm{ol} .2014 .2715$

\begin{abstract}
Only a few previous studies have demonstrated an association between resistance to thyroid hormone (RTH) and thyroid cancer. The current study presents the case of a 67-year-old female who was referred to the Third Xiangya Hospital of Central South University with an enlargement of the neck that had grown gradually over two years and subsequently, rapidly enlarged over the two months prior to admission, alongside a slight sensation of shortness of breath. Laboratory data revealed a significantly increased level of thyroid-stimulating hormone (TSH), total triiodothyronine, total thyroxine, free triiodothyronine, free thyroxine, thyroprotein and thyroglobulin antibody; however, the levels of thyroperoxidase and TSH receptor antibody were within the normal ranges. A thyroid hormone suppression test revealed a TSH reduction of 32\%, Magnetic resonance imaging of the pituitary gland was negative for abnormalities. The patient's thyroid pathology revealed a non-Hodgkin's lymphoma of the thyroid. CHOP + nimustine chemotherapy significantly reduced the clinical symptoms. The genetic analysis revealed a novel point mutation of the thyroid hormone receptor $\beta$ (THRB) gene in exon 10 (g1680 G to A) in the 3'-untranslated region of the patient. To the best of our knowledge, this is the first case report of RTH with thyroid non-Hodgkin's lymphoma, which involved a mutation (g1680 G to A) in exon 10 of THRB.
\end{abstract}

\section{Introduction}

Resistance to thyroid hormone (RTH), also known as Refetoff syndrome, is a rare syndrome that manifests as reduced end-organ responsiveness to the thyroid hormone. The precise incidence of RTH is unclear. A study observed that high blood T4 levels were present in one case per 40,000 in neonatal

Correspondence to: Dr Zhaohui Mo, Department of Endocrinology, Third Xiangya Hospital of Central South University, 138 Tong Zi Po Street, Changsha, Hunan 410013, P.R. China E-mail: easd04mzh@126.com

Key words: thyroid resistance syndrome, thyroid non-Hodgkin's lymphoma, thyroid hormone receptor $\beta$, mutation screening (1). Patients with RTH exhibit elevated serum free thyroxine (FT4), free triiodothyronine (FT3) and normal or elevated serum thyroid stimulating hormone (TSH) levels. The characteristic clinical features vary, including an absence of the usual symptoms of hyperthyroidism/hypothyroidism, hyperthyroidism or hypothyroidism, with or without goiter (2). The majority of cases are related to thyroid hormone receptor $\beta$ (THRB) mutations, a few cases are caused by thyroid hormone receptor $\alpha$ (THRA) mutations, and even fewer cases have no THR mutation, which may be associated with post transcriptional regulation (3-8).

Primary thyroid lymphoma (PTL) is a rare form of thyroid cancer, it accounts for 1-5\% of all thyroid malignancies and $1-2 \%$ of all extra-nodal lymphomas. Typically patients present with a rapidly enlarging thyroid as opposed to other thyroid malignancies, about $30-50 \%$ of patients have complications with hoarseness, stridor, dysphagia and a pressure sensation in the neck (9).

Recent studies have reported that RTH is associated with certain types of thyroid cancer, including papillary thyroid carcinoma and papillary microcarcinoma (10-14). In the current study, we report a case of RTH with thyroid non-Hodgkin's lymphoma.

\section{Case report}

Written informed consent was obtained from the patient and the patient's family. A 67-year-old female was referred to the Third Xiangya Hospital of Central South University (Changsha, China) in December 2012 with a neck that had become gradually enlarged over the previous two years, with rapid enlargement in the previous two months, accompanied by a slight sensation of shortness of breath (Fig. 1). During the two years, no hyperthyroidism or hypothyroidism symptoms such as sensitivity to heat, irritability, tremors or sensitivity to the cold, fatigue and edema were experienced. No history of irradiation or family history of thyroid disease was reported. On admission, pulse rate was $82 \mathrm{bpm}$, regular blood pressure was $145 / 59 \mathrm{mmHg}$ and body temperature was $36.6^{\circ} \mathrm{C}$. Physical examination revealed a third degree enlargement of the left lateral lobe of the thyroid; the right lateral lobe thyroid was normal and no proptosis was present. Laboratory investigations revealed significantly elevated levels of serum TSH [33.63 $\mu \mathrm{IU} / \mathrm{ml}$; normal range $(\mathrm{N})$, 0.27-4.2], total T3 (TT3; $3.11 \mathrm{nmom} / 1$; N, 1.3-3.1), total T4 (TT4; 


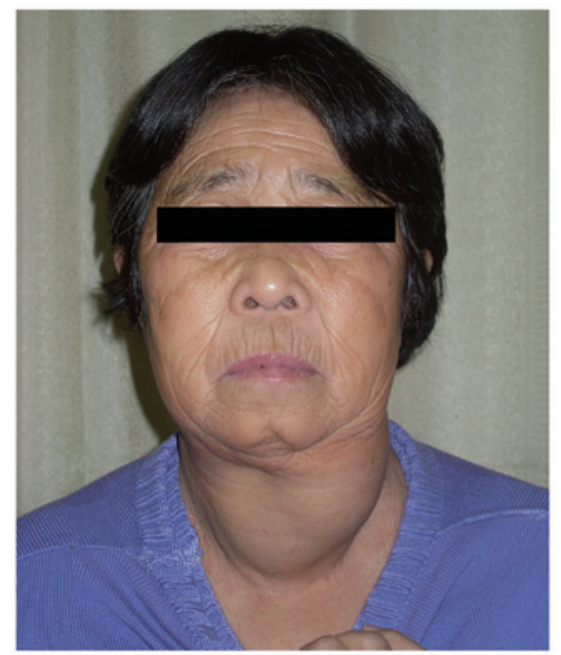

Figure 1. Physical examination revealed a third degree enlargement of the left lateral lobe of the thyroid. The right lateral lobe of the thyroid was normal.
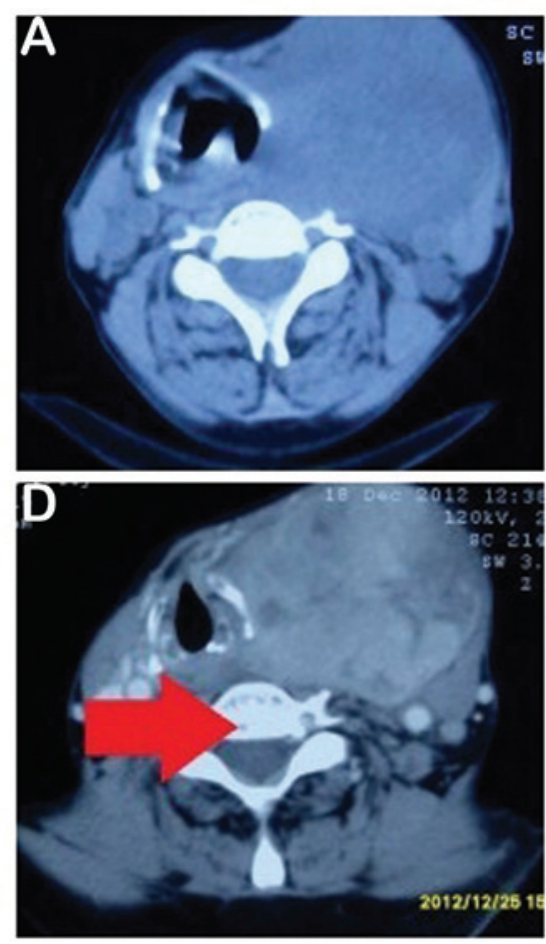
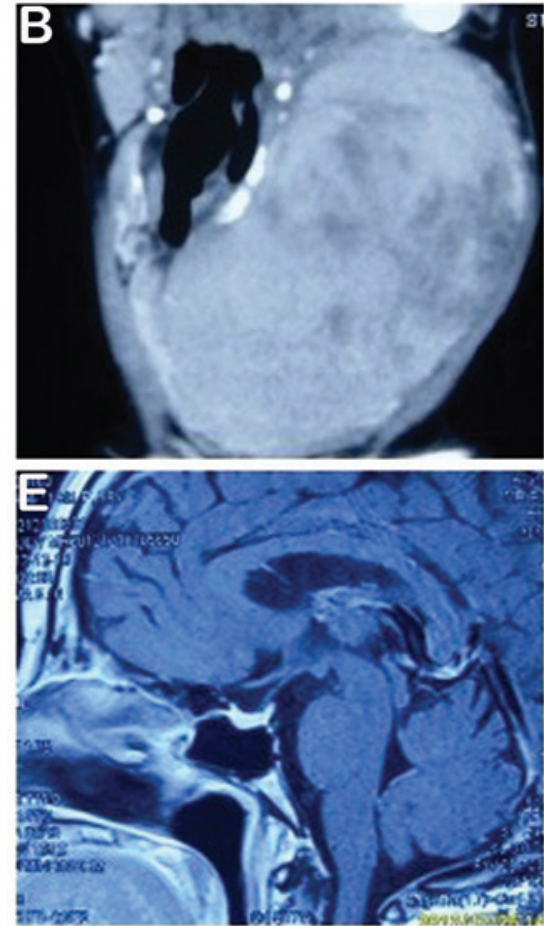
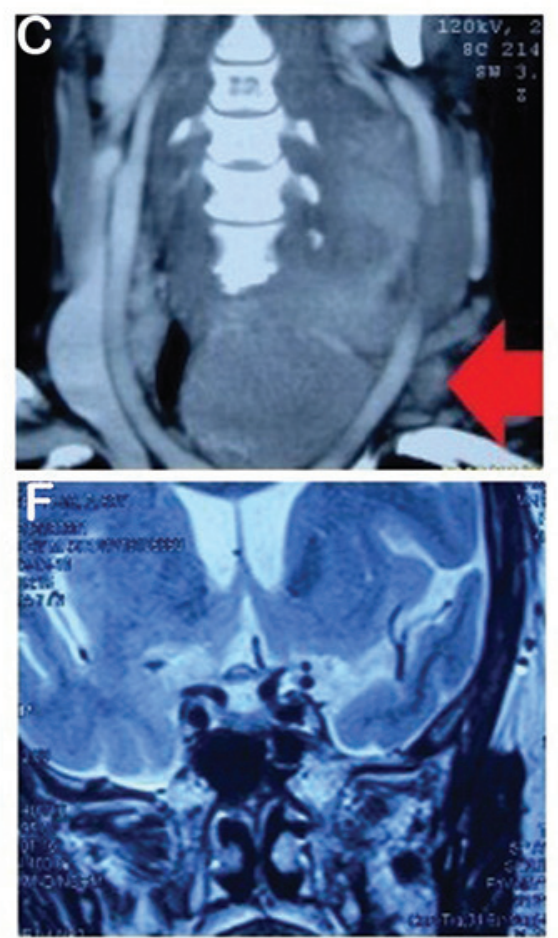

Figure 2. Neck CT and pituitary MRI images of the patient. Cervical CT revealed a heterogeneous mass of $7.3 \times 5.3 \times 9.2 \mathrm{~cm}$ in size in the left lateral lobe of the thyroid gland, a tracheal compression and shift to the right, lumen narrowing and multiple irregular low-density areas in mass (CT value about 44-100 HU). The right side of the thyroid was a normal size, with low density nodules. Neck CT images: (A) horizontal plane, (B) sagittal plane, (C) coronal plane (upper mediastinal lymph node enlargement, arrow head) and (D) cervical circular low density shadow (arrow head).Pituitary MRI of the patient did not reveal any pathological findings in either the (E) sagittal or (F) coronal planes. CT, computed tomography; MRI, magnetic resonance imaging.

$>320 \mathrm{nmom} / \mathrm{l} ; \mathrm{N}, 66-181$ ), thyroperoxidase (TPO) antibody (23.8 IU/ml; N, 0-34), TSH receptor antibody (TRAB; <0.3 IU/l; $\mathrm{N}, 0-1.75)$. In the outpatient clinic the following day, the thyroid hormone examination was repeated, yielding serum TSH values of $32.28 \mu \mathrm{IU} / \mathrm{ml}$, TT3 of $3.58 \mathrm{nmom} / 1$, TT4 of more than $320 \mathrm{nmom} / 1$, free T3 (FT3) of $9.57 \mathrm{pmom} / \mathrm{l}$ (N, 3.1-6.8 pmom/l), free T4 (FT4) of >100 pmom/1 (N, 12-22 pmom/l), TPO antibody of $13.56 \mathrm{IU} / \mathrm{ml}$, TRAB of $1.3 \mathrm{IU} / \mathrm{l}$, thyroglobulin antibody (TgAB) of $141.2 \mathrm{IU} / \mathrm{ml}(\mathrm{N}, 0-115)$, thyroprotein $(\mathrm{TG})$ of $192.3 \mathrm{ng} / \mathrm{ml}$ (N, 1.4-7.8). Blood cell count showed a white blood cell level of $13.8 \times 10^{9} / 1$ (N, 4.0-10.0), hemoglobin level of $92 \mathrm{~g} / \mathrm{l}$ (N, 110-150), platelet level of $233 \times 10^{9} / 1(\mathrm{~N}, 100-300)$ and serum albumin of $34.2 \mathrm{~g} / \mathrm{l}$ (N, 35.0-50.0). The thyroid color Doppler ultrasound scan revealed a hypoechoic mass on the left lateral lobe of the thyroid, while the right lateral lobe of the thyroid had an uneven echo. The additional color Doppler ultrasound results of the liver, gallbladder, pancreas, spleen, retroperitoneal lymph node, uterus and ovary, kidney, ureter, bladder and bilateral adrenal were all normal. A chest X-ray revealed a widened mediastinum, tracheal compression and cardiac 


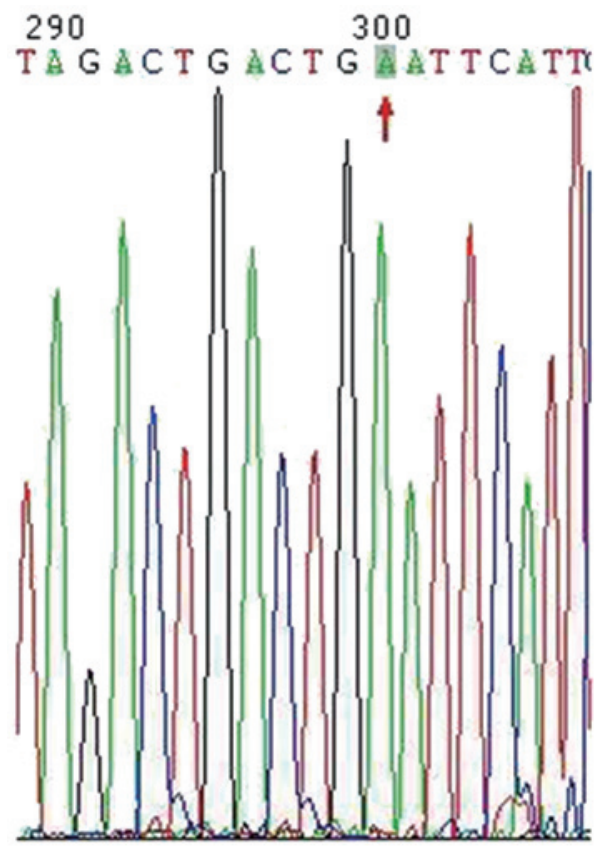

Figure 3. Sequence analysis of the thyroid hormone receptor $\beta$ gene showing a 3'-UTR mutation (g1680 G to A) at exon 10 (red arrow). The same mutation was observed in the patient's son and daughter.

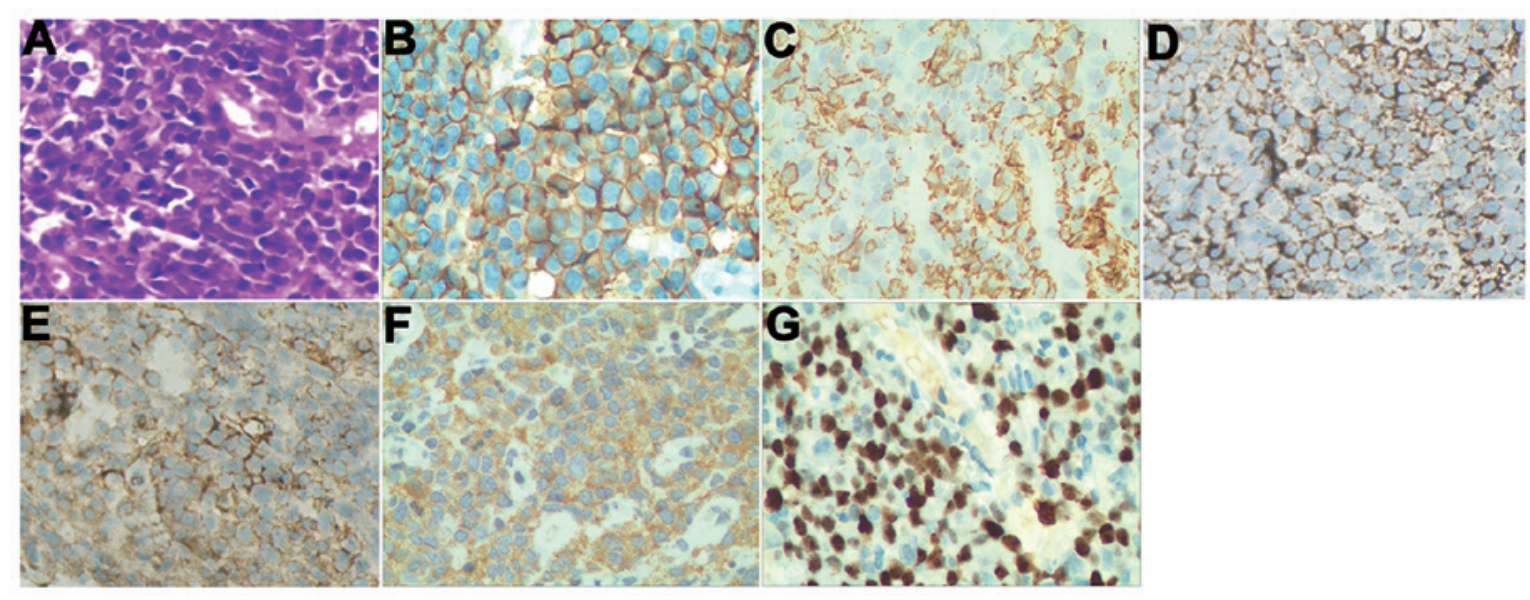

Figure 4. Tissue pathology staining was positive for B-cell and plasma cell surface markers. (A) Hematoxylin and eosin staining, and positive staining for (B) CD38, (C) CD20, (D) Kappa chain, (E) lambda chain, (F) Bcl-2 and (G) Ki 67.

enlargement (primarily an enlarged left ventricle). A cervical computed tomography (CT) showed a thyroid left lateral lobe tumor, considered to be thyroid cancer and possible bilateral neck metastases, multiple mediastinal lymph node metastases and cervical vertebra centrum bone shifts (Fig. 2A-D). Single photon emission computed tomography (SPECT) of the thyroid showed that the volume of the left lateral lobe had increased significantly and there were multiple bilateral thyroid nodules. Therefore, the possibility of a malignant tumor was considered. Bone marrow cytology examination showed obviously active bone marrow hyperplasia and normal cells in different stages. Magnetic resonance imaging (MRI) of the pituitary did not reveal any pathological findings (Fig. 2E-F).

A dexamethasone suppression test ( $2 \mathrm{mg} \mathrm{q} 6 \mathrm{~h}$ for two days) showed that the level of TSH had decreased from 35.53 to $16.3 \mu \mathrm{IU} / \mathrm{ml}$, a thyroid hormone suppression test (thyroid tablets $100 \mathrm{mg}$ qd for two days) revealed that the TSH had decreased from $32.58 \mu \mathrm{IU} / \mathrm{ml}$ to $18.26 \mu \mathrm{IU} / \mathrm{ml}$. It was not possible to measure L-T3.

The genetic analysis presented in Fig. 3 revealed that exons 1-9 were normal, and that exon 10 had a novel point mutation of THRB ( $\mathrm{g} 1680 \mathrm{G}$ to A); the same mutation was identified in the patient's son and daughter. To exclude the mutation as a THRB polymorphism, the sequences of 100 healthy controls were analyzed and the mutation was not found (data not shown). A core biopsy, stained with hematoxylin and eosin (HE), showed round and polygon poorly differentiated tumor cells (Fig. 4A). The results of immunohistochemical staining were as follows: CD20 (+), CD38 (+), immunoglobulin light chain [Kappa (+) and Lambda (+)], BCL-2 (+), CD79 $\alpha(-)$, CD34 (-), CD3 (-), CD5 (-), CD10 (-), CKPan (-), EMA (-), CD30 (-) and TdT (-) (Fig. 4). Ki67 stained $\sim 40 \%$ of the cells. 
These results indicated a non-Hodgkin's lymphoma [lymphoplasmacytic lymphoma (LPL)]. The patient's serum showed an IgA level of $1.05 \mathrm{~g} / \mathrm{l}(\mathrm{N}, 0.5-5 \mathrm{~g} / \mathrm{l})$, an IgE level of $31 \mathrm{IU} / \mathrm{ml}$ (N, 0-385 IU/ml), an IgG level of 7.8 g/1 (N, 5.16-14.25 g/l) and an IgM level of $1.13 \mathrm{~g} / \mathrm{l}(\mathrm{N}, 0.3-2.09 \mathrm{~g} / \mathrm{l})$; no M protein was identified by protein electrophoresis.

The patient was transferred to the department of hematology for CHOP + nimustine chemotherapy, and, following a course of treatment, the neck swelling was significantly reduced and shortness of breath was improved. The patient and son refused the subsequent treatment and the collection of blood samples from other family members, therefore no clinical and genetic data could be obtained for the family.

\section{Discussion}

Due to a low morbidity and complicated clinical manifestations, RTH is often misdiagnosed. Prior to diagnosis of RTH, alternative causes of elevated TT4 and TSH must be excluded, for example, raised serum binding proteins, non-thyroidal illness (including acute psychiatric disorders), drug use (amiodaron, heparin), familial dysalbuminemic hyperthyroxinemia and TSH secreting pituitary adenoma (TSH-oma); the most difficult distinction is between RTH and TSH-oma (15). In the current case, non-thyroidal illness, increased albumin and drugs were excluded. The patient's TT4, TG and TGA levels were significantly increased, however, the levels of FT3 and FT4 were also increased, which aided in excluding elevated TT4 due to increased serum TGB. A thyroid suppression test showed that $\mathrm{TSH}$ was reduced by $32 \%$, pituitary MRI was negative and further the genetic analysis supported the view that the patient had RTH.

RTH is a rare autosomal, hereditary disease, consisting of $75-85 \%$ familial cases and $15-25 \%$ sporadic cases, with THRB mutations causing RTH in $\sim 90 \%$ of cases (16). In the current study, the patient, and the patient's son and daughter all possessed a novel point mutation in exon 10 (g1680 G to A) of THRB. The point mutation was located in the 3'-untranslated region (UTR) rather than in the coding sequence (CDS), however, this mutation may affect the post-transcription processing of THRB.

Patient pathology revealed that this case did not belong to the most common type of PTL, rather, it was indicated to be a LPL (17). LPL is an extremely rare subtype of non-Hodgkin's lymphoma, which is a low-grade, B-cell neoplasm composed of small lymphocytes, plasmacytoid lymphocytes and plasma cells that typically involve the bone marrow, lymph node or spleen. However, a few cases do not originate in the bone marrow but are extramedullary. If the extramedullary tumor infiltrates bone marrow, it may cause pancytopenia, organomegaly and hyperviscosity; the majority of cases are asymptomatic or present with anemia. The pathological features usually present as eccentric nuclei and a more abundant basophilic cytoplasm. The typical immunophenotype of LPL shows expression of CD19, CD20, CD22, FMC7, BCL2, CD38 and CD79a; however, CD5, CD10, and CD23 are usually absent (18). In the current case a primary tumor was not derived from bone marrow and non-Hodgkin's lymphoma did not infiltrate the bone marrow; although the CT scan indicated possible cervical bone metastases, only mild anemia was detected, the bone marrow cytology did not suggest lymphocyte and plasma cell infiltration. Additionally, M protein was not detected and the $\mathrm{Ig} \mathrm{M}$ level was within the normal range. The immunohistochemical staining supported the diagnosis of thyroid LPL (10-14).

A number of studies of RTH with thyroid cancer have been previously reported (10-14). In an animal study, mutation of THRB significantly increased the morbidity of spontaneous thyroid carcinoma through the activation of TSH-mediated signaling pathways (19). Whether or not the mutation of THRB increases the morbidity of non-Hodgkin's lymphoma or LPL remains unclear.

In conclusion, to the best of our knowledge this is the first report of a case of RTH with thyroid non-Hodgkin's lymphoma. The pathology may be considered as LPL, which is an extremely rare subtype of non-Hodgkin's lymphoma. The present case indicated that the point mutantion of THRB in 3'-UTR might be an important indicator for RTH or non-Hodgkin's lymphoma. Further functional studies will be performed in order to confirm the function of this THRB mutation in RTH or non-Hodgkin's lymphoma.

\section{References}

1. Lafranchi SH, Snyder DB, Sesser DE, et al: Follow-up of newborns with elevated screening T4 concentrations. J Pediatr 143: 296-301, 2003.

2. Olateju TO and Vanderpump MP: Thyroid hormone resistance. Ann Clin Biochem 43: 431-440, 2006

3. Zavacki AM and Larsen PR: RTHalpha, a newly recognized phenotype of the resistance to thyroid hormone $(\mathrm{RTH})$ syndrome in patients with THRA gene mutations. J Clin Endocrinol Metab 98: 2684-2686, 2013.

4. Schoenmakers N, Moran C, Peeters RP, Visser T, Gurnell M and Chatterjee K: Resistance to thyroid hormone mediated by defective thyroid hormone receptor alpha. Biochim Biophys Acta 1830: 4004-4008, 2013.

5. Pohlenz J, Weiss RE, Macchia PE, et al: Five new families with resistance to thyroid hormone not caused by mutations in the thyroid hormone receptor beta gene. J Clin Endocrinol Metab 84: 3919-3928, 1999.

6. Fozzatti L, Lu C, Kim DW, et al: Resistance to thyroid hormone is modulated in vivo by the nuclear receptor corepressor (NCOR1). Proc Natl Acad Sci USA 108: 17462-17467, 2011.

7. Bottcher Y, Paufler T, Stehr T, Bertschat FL, Paschke R and Koch CA: Thyroid hormone resistance without mutations in thyroid hormone receptor beta. Med Sci Monit 13: CS67-CS70, 2007.

8. Işık E, Beck Peccoz P, Campi I, et al: Thyroid hormone resistance: a novel mutation in thyroid hormone receptor beta (THRB) genecase report. Turk J Pediatr 55: 322-327, 2013.

9. Walsh S, Lowery AJ, Evoy D, McDermott EW and Prichard RS: Thyroid lymphoma: recent advances in diagnosis and optimal management strategies. Oncologist 18: 994-1003, 2013.

10. Unluturk U, Sriphrapradang C, Erdogan MF, et al: Management of differentiated thyroid cancer in the presence of resistance to thyroid hormone and TSH-secreting adenomas: a report of four cases and review of the literature. J Clin Endocrinol Metab 98: 2210-2217, 2013

11. Ramos-Prol A, Antonia Perez-Lazaro M, Isabel del Olmo-Garcia M, et al: Differentiated thyroid carcinoma in a girl with resistance to thyroid hormone management with triiodothyroacetic acid. J Pediatr Endocrinol Metab 26: 133-136, 2013.

12. Paragliola RM, Lovicu RM, Locantore P, et al: Differentiated thyroid cancer in two patients with resistance to thyroid hormone. Thyroid 21: 793-797, 2011.

13. Sugita M, Harada $H$ and Yamamoto T: Perioperative management of a patient with thyroid hormone resistance who underwent total thyroidectomy for thyroid cancer. J Anesth 26: 595-597, 2012.

14. Kim HK, Kim D, Yoo EH, et al: A case of resistance to thyroid hormone with thyroid cancer. J Korean Med Sci 25: 1368-1371, 2010 . 
15. Agrawal NK, Goyal R, Rastogi A, Naik D and Singh SK: Thyroid hormone resistance. Postgrad Med J 84: 473-477, 2008.

16. Takeda K, Sakurai A, DeGroot LJ and Refetoff S: Recessive inheritance of thyroid hormone resistance caused by complete deletion of the protein-coding region of the thyroid hormone receptor-beta gene. J Clin Endocrinol Metab 74: 49-55, 1992.

17. Naderi $\mathrm{N}$ and Yang DT: Lymphoplasmacytic lymphoma and Waldenstrom macroglobulinemia. Arch Pathol Lab Med 137: 580-585, 2013.
18. Lin P, Molina TJ, Cook JR and Swerdlow SH: Lymphoplasmacytic lymphoma and other non-marginal zone lymphomas with plasmacytic differentiation. Am J Clin Pathol 136: 195-210, 2011.

19. Zhao L, Zhu X, Won Park J, Fozzatti L, Willingham M and Cheng SY: Role of TSH in the spontaneous development of asymmetrical thyroid carcinoma in mice with a targeted mutation in a single allele of the thyroid hormone-beta receptor. Endocrinology 153: 5090-5100, 2012. 